\title{
A new look at a polar crown cavity as observed by SDO/AIA
}

\section{Structure and dynamics ${ }^{\star}$}

\author{
S. Régnier, R. W. Walsh, and C. E. Alexander
}

Jeremiah Horrocks Institute, University of Central Lancashire, Preston, Lancashire, PR1 2HE, UK

e-mail: SRegnier@uclan.ac.uk

Received 31 May 2011 / Accepted 21 July 2011

\begin{abstract}
Context. The Solar Dynamics Observatory (SDO) was launched in February 2010 and is now providing an unprecedented view of the solar activity at high spatial resolution and high cadence covering a broad range of temperature layers of the atmosphere. Aims. We aim at defining the structure of a polar crown cavity and describing its evolution during the erupting process.

Methods. We use the high-cadence time series of SDO/AIA observations at $304 \AA$ (50000 K) and $171 \AA$ ( $0.6 \mathrm{MK})$ to determine the structure of the polar crown cavity and its associated plasma, as well as the evolution of the cavity during the different phases of the eruption. We report on the observations recorded on 13 June 2010 located on the north-west limb.

Results. We observe coronal plasma shaped by magnetic field lines with a negative curvature (U-shape) sitting at the bottom of a cavity. The cavity is located just above the polar crown filament material. We thus observe the inner part of the cavity above the filament as depicted in the classical three part coronal mass ejection (CME) model composed of a filament, a cavity, and a CME front. The filament (in this case a polar crown filament) is part of the cavity, and it makes a continuous structuring from the filament to the CME front depicted by concentric ellipses (in a 2D cartoon).

Conclusions. We propose to define a polar crown cavity as a density depletion sitting above denser polar crown filament plasma drained down the cavity by gravity. As part of the polar crown filament, plasma at different temperatures (ranging from $50000 \mathrm{~K}$ to $0.6 \mathrm{MK}$ ) is observed at the same location on the cavity dips and sustained by a competition between the gravity and the curvature of magnetic field lines. The eruption of the polar crown cavity as a solid body can be decomposed into two phases: a slow rise at a speed of $0.6 \mathrm{~km} \mathrm{~s}^{-1}$ and an acceleration phase at a mean speed of $25 \mathrm{~km} \mathrm{~s}^{-1}$.
\end{abstract}

Key words. Sun: corona - Sun: coronal mass ejections (CMEs) - Sun: filaments, prominences - Sun: UV radiation

\section{Introduction}

Launched in February 2010, the Solar Dynamics Observatory (SDO) is the first NASA Living With a Star mission. On board SDO has three different instruments dedicated to studying the magnetic and plasma evolution of the solar corona, its associated eruptive events, and their consequences on the Earth. Here we analyse observations from the Atmospheric Imager Assembly (AIA) with high time cadence $(\sim 12 \mathrm{~s})$ and spatial resolution $\left(\sim 1^{\prime \prime}\right)$, which provide much more detail of the corona on the limb than other comparable instruments, such as SOHO/EIT and STEREO/SECCHI/EUVI. It has been shown that SDO/AIA has the sensitivity to observe far more off-limb structures than ever before (Lemen et al. 2011). Here we report on one of these structures: a cavity observed on 13 June 2010 associated with an erupting polar crown filament/prominence leading to a propagating coronal mass ejection (CME) at a speed of about $300-350 \mathrm{~km} \mathrm{~s}^{-1}$ (as reported in the SOHO/LASCO CME catalogue, http: //cdaw.gsfc.nasa.gov/CME_list/).

Typically CMEs are observed in white light coronograph with a three-part structure: the bright core related to the filament material, the cavity surrounding the core, and the bright front of the CME marking the transition between the CME and the ambient corona (see e.g., Illing \& Hundhausen 1986). In a recent paper by Gibson et al. (2010), the authors studied the structure,

* Two movies are only available at http://www . aanda.org shape, and evolution (mostly due to the solar rotation) in detail of a stable cavity observed by SOHO/EIT in the wavelength filter at $195 \AA$. They find that, based on a forward modelling approach, the cavity is darker than the surrounding owing to the depletion in density by a factor of about 2 . It is clear in their study that the prominence cavity analysed corresponds to the classical cavity as defined in the three-part CME model mentioned above.

The formation and instability of cavities associated with a CME have been extensively modelled (see reviews by Lin 2002; Forbes et al. 2006). The structure often contains a twisted flux tube able to accumulate magnetic energy and mass within it, which thus can be destabilised by catastrophic mechanisms and/or external triggers. Despite several studies of the thermal structures of cavities especially from white-light coronographs, eclipse observations, EUV, and soft X-ray imaging (e.g., Hudson et al. 1999; Hudson \& Schwenn 2000; Gibson et al. 2006; Habbal et al. 2010), there is to our knowledge no observational evidence of a long time series and of high-cadence obsevations of cavity at different temperatures as provided by SDO/AIA data able to (i) clearly demonstrate the thermal structure of both the prominence and cavity material, and (ii) describe how the plasma of a polar-crown filament evolves before and during the eruption.

Thus, the observations described here focus for the first time on the dynamics of the inner part of the cavity above the polar crown filament/prominence material and its evolution during the eruptive phase. We first describe the multithermal structure of 
(a)
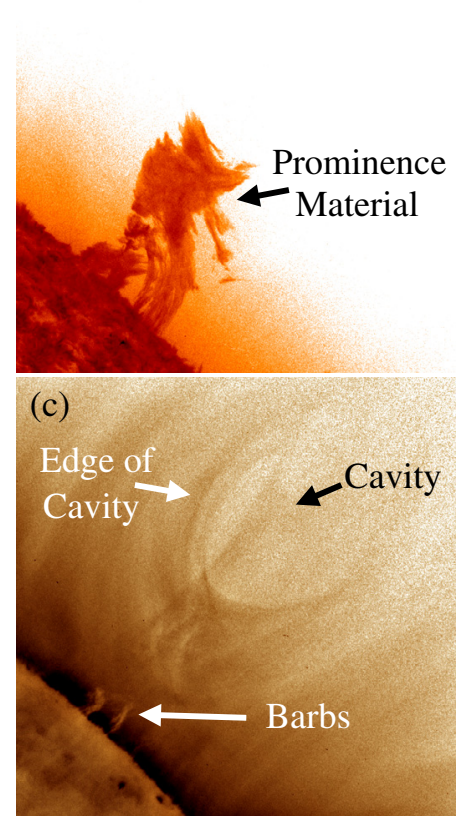

(b)

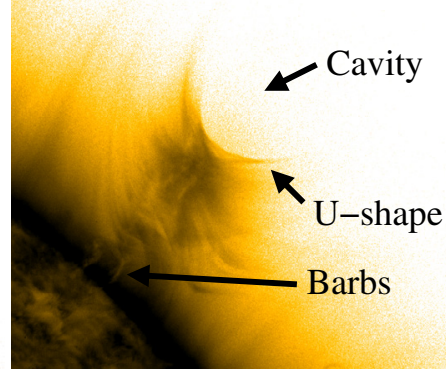

(d)

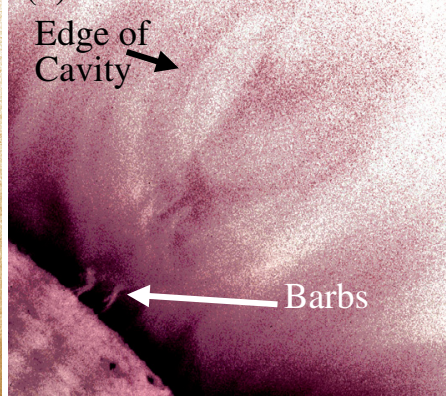

Fig. 1. Off-limb close-up on the cavity structure as observed by SDO/AIA at $304 \AA$ (left) and at $171 \AA$ (right) at 03:24:12 UT (negative images).

the polar crown filament/cavity (Sect. 2) and then the evolution of the plasma during the eruption in two different wavelengths (Sect. 3). In Sect. 4, we propose a definition for a polar crown cavity and discuss the implications of our study on eruptive filament models.

\section{Multithermal observations of the cavity}

The event was observed on 13 June 2010 between 00:00 and 12:00 UT on the north-west limb. We focus only on the data provided by the SDO/AIA instrument using the full spatial resolution and a reduced time cadence of three minutes (instead of the nominal $12 \mathrm{~s}$ ). The SDO/AIA data are processed at level 1 (test series), which includes removal of bad pixels and spikes. The time series were corrected for pointing and jitter effects. The image calibration corresponds to a first approximation but does not influence our study.

An overview of the structures observed by SDO/AIA is given in Fig. 1 at different temperatures: (a) HeII at $304 \AA$ at about $50000 \mathrm{~K}$, (b) FeIX at $171 \AA$ at about 0.6 MK, (c) FeXII at $193 \AA$ at about 1.6 MK (with a hot contribution of FeXXIV at $2 \mathrm{MK}$ ), and (d) FeXIV at $211 \AA$ at about $2 \mathrm{MK}$. The single temperature associated with each channel corresponds to the main peak of emission in the temperature response functions provided by the SDO/AIA team (Lemen et al. 2011). The study of the SDO/AIA channel thermal response performed by O'Dwyer et al. (2010) shows the properties of the different SDO/AIA broadband channels in different regions of the Sun (active region, quiet Sun, and coronal hole). The prominence and cavity material is supposed to be at or near the temperatures mentioned above. The observed structures are

(i) cool and hot plasma off the limb, which are parts of a polar crown filament (see Figs. 1a,b). The plasma is confined in an area with a characteristic height of $100 \mathrm{Mm}$ and width of $80 \mathrm{Mm}$ (at the start of the time series); (ii) a dark cavity seen as a complete ellispe in the $193 \AA$ and $211 \AA$ channels (see Figs. 1c,d);

(iii) elongated barbs seen as dark material in the hot channels and connecting the photosphere/chromosphere regions to the cavity (and/or the bottom of the cavity) to supply or evacuate mass from the filament. We do not discuss the dynamics of the barbs and their implications in the eruption process in this letter.

Figures $1 \mathrm{a}$ and $\mathrm{b}$ show the co-spatiality of the polar-crown material in both the $304 \AA$ and $171 \AA$ channels. These snapshots highlight

- the upward (U-shaped) bending of magnetic field lines at the bottom of the inner cavity; and

- the accumulation of prominence material along these field lines suggesting that a magnetohydrostatic equilibrium is in place with the magnetic field curvature acting against the gravity.

The latter assumption is also supported by the fact that the cavity has been stable for several hours before the eruption. Even if the location is similar, the U-shaped field lines in both channels are not filled by the corresponding plasma in the same manner: in the $171 \AA$ channel the length along the U-shaped field lines filled by the coronal plasma appears longer than in the $304 \AA$ channel.

It is important to remember here that the observed structure is integrated along the line-of-sight. Therefore limited threedimensional depth information can be derived on the polar crown filament solely from this SDO/AIA dataset.

\section{Evolution of the cavity}

To study the dynamics of the eruption, we first looked at several snapshots of the cavity to describe the motions and structures of the polar crown filament. We have restricted the dynamical study to the $304 \AA$ and $171 \AA$ channels in which the U-shaped structures are clearly seen and also for which there is a minimum of confusion with the background emission (see Figs. 1c,d).

Figure 2 outlines a series of images at four characteristic times of the cavity evolution: (a) at 00:03 UT when the cavity is stable at a height of $100 \mathrm{Mm}$ above the surface (projection on the plane of the sky), (b) at 03:24 UT in the early phase of the eruption, (c) at 06:51 UT towards the end of cavity eruption within the SDO/AIA field-of-view, and (d) at 09:00 UT, a couple of hours after the cavity has moved into the higher part of the corona, and the plasma and magnetic field lines are still in the process of reorganisation and relaxation.

The polar crown material is divided into two parts, namely P1 and P2, which are not distinguishable at first (Fig. 2a) but can be differentiated in the following frames (Figs. 2b,c by the solid line parallel to the limb). P1 corresponds to the main part of the eruptive cavity. In Fig. 2b, the plasma contained in U-shaped fied lines starts to move upwards (as indicated by the left arrow), whilst the plasma on the right-hand side exhibits upwards flows along field lines (right arrow). P2 remains stable. In Fig. 2c, the plasma in P1 is detached and thus ejected into the high corona. $\mathrm{P} 2$ starts to rise.

In Fig. 2d, only the plasma in $\mathrm{P} 2$ remains at this height in the corona, whilst P1 continues its way out of the corona. The plasma in P2 is flowing down towards the low corona following the field lines in both channels (see arrows). From this time series, it is important to notice that the plasma in both EUV channels are located at the same place below the polar crown cavity, 
(a)

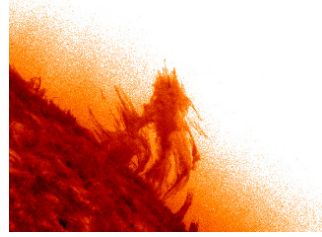

(c)

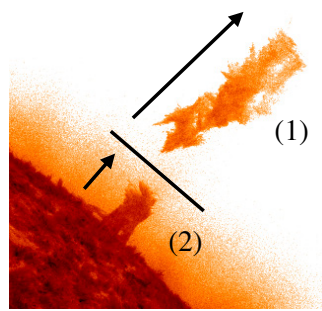

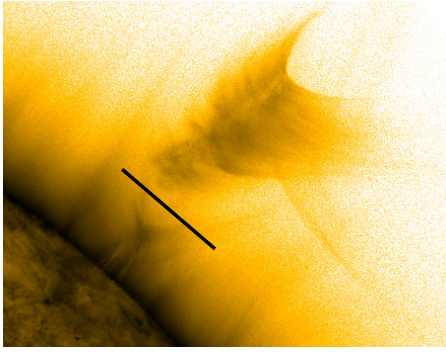

(b)

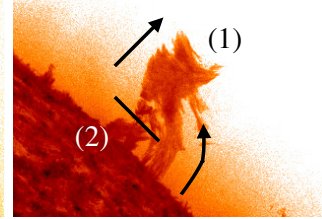

(d)

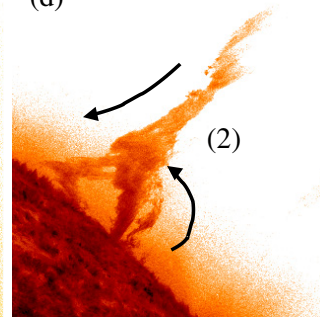

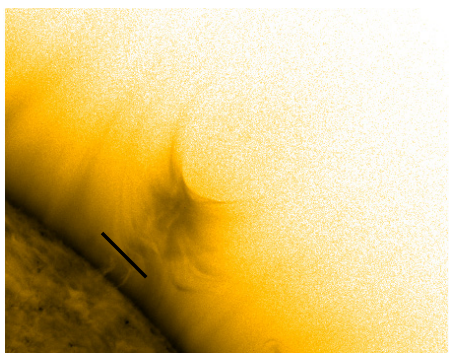

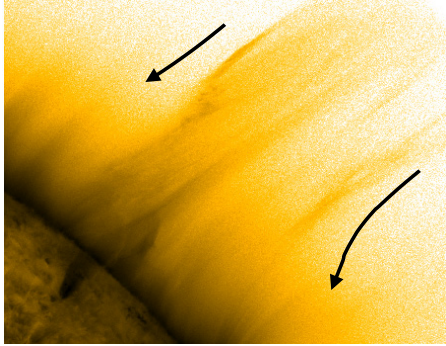

Fig. 2. Evolution of the polar crown cavity and the associated plasma (left) at $50000 \mathrm{~K}(304 \AA)$ and (right) at $0.6 \mathrm{MK}(171 \AA)$ at three different times: a) 00:03:12 UT, b) 03:24:11 UT, c) 06:51:11 UT, and d) 09:00:11 UT. The white arrows indicate the direction of the plasma motions. The lines parallel to the solar limb divide the different parts of plasma involved in the eruption (see text).
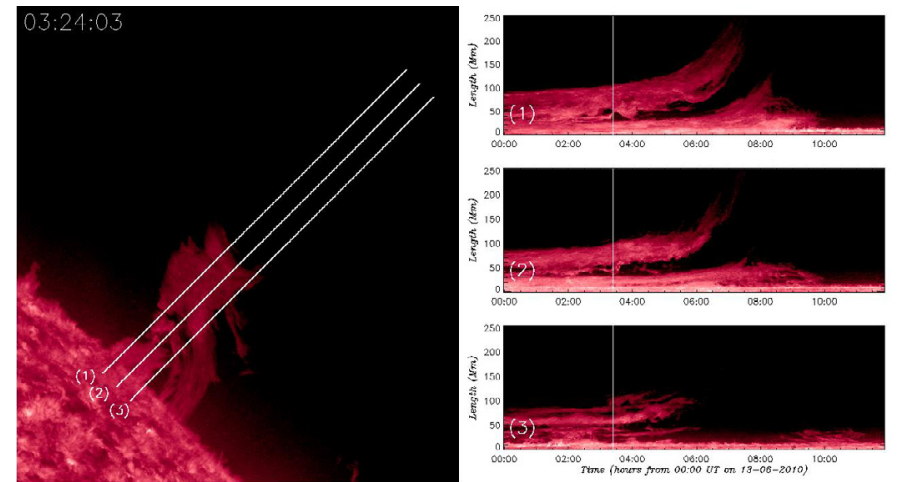

Fig. 3. Time slices of the eruptive cavity as observed in $304 \AA$ on 13 June 2010 by SDO/AIA. The image on the left has been taken at 03:24:12 UT in log scale to highlight the cavity. On the right, the time slices are plotted along three different radial directions: (1) and (3) are on the sides of the cavity, (2) is at the minimum of the cavity. The three locations are chosen at the beginning of the time series running from 00:00 UT to 12:00 UT. The vertical axis represents the distance in Mm from the bottom (on the disc) of the time slices. The white vertical line indicates the time of the image on the left. The movie is provided as online material.

and it is only during the eruptive phase that the decoupling between the two is observed.

Second, we examined the radial evolution of the cavity at three different locations along the width of the cavity by plotting three adjacent time slices (Figs. 3 and 4). The middle location (2) corresponds to the minimum height of the cavity above the surface at the beginning of the time series, whilst locations (1) and (3) are symmetrical on both sides of the minimum height. The cavity appears in the top left-hand corner of the time slices, and the bottom of the cavity is first located at about $90 \mathrm{Mm}$ at $304 \AA$ and $100 \mathrm{Mm}$ at $171 \AA$. Even if the motion of the cavity is not entirely in the radial direction, the three time slices show the behaviour of a large portion of the cavity during the eruption. The similar evolution in all three slices supports the assumption that the cavity evolves as a solid body. We note that the cavity leaves the field-of-view (250 Mm high) at about the same time in both wavelengths (around 07:00 UT).
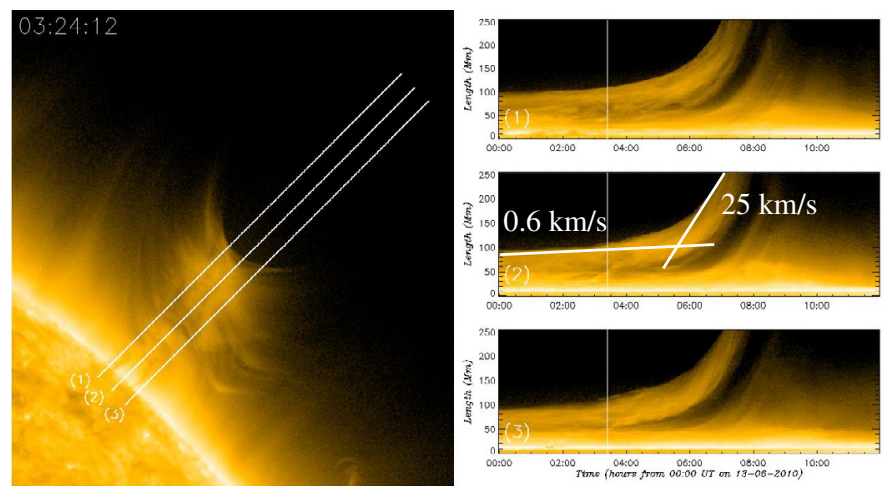

Fig. 4. Same as Fig. 3 for the $171 \AA$ A channel. The asymptotic behaviour is indicated by the straight white lines. The estimated speeds are also indicated. The movie is provided as online material.

In Fig. 3, the time slices show the evolution of the cool plasma at $304 \AA$ during the eruption process: the slow rise of the cavity during four to five hours and then the eruption. The plasma of the cavity observed at $304 \AA$ does not reach heights above $250 \mathrm{Mm}$. During the eruption process, most of the plasma is drained back down along magnetic structures as highlighted in Fig. 2.

From Fig. 4, we define the initial and final stages of the eruption as observed at $171 \AA$ from the asymptotic behaviour of the time slice (2) as indicated by the straight white lines. At the start, the cavity is stable, justifying starting our study at 00:00 UT. The first phase is a slow rise of the cavity with a characteristic speed of $0.6 \mathrm{~km} \mathrm{~s}^{-1}$. The cavity follows this trend until 03:00 UT. The second phase of the eruption is the faster motion of the cavity with a characteristic speed of $25 \mathrm{~km} \mathrm{~s}^{-1}$. Even if this speed is far less than local Alfvén or sound speeds, this is comparable to the speed of plasmoid ejection as reported by Tsuneta (1997).

\section{Discussion and conclusions}

We propose to define a polar crown cavity as a density depletion at the bottom of which the polar crown filament material sits, thereby indicating the existence of a magnetohydrostatic 
equilibrium. The filament material is drained down along the polar crown cavity by gravity and sustained by the action of the upward-directed magnetic field curvature force. This fact, as well as the long, steady observations of the polar crown cavity, indicates that the cavity is in a magnetohydrostatic equilibrium. The cold and hot coronal plasma are located at a similar location along the same field lines. The observations of the cavity structure and plasma spatial distribution are consistent with the classical 2D cartoon of a cavity depicted by concentric ellipses. For instance, in the classical CSHKP model (Carmichael 1964; Sturrock 1968; Hirayama 1974; Kopp \& Pneuman 1976), the eruptive structure is composed of a twisted flux tube at the bottom of which the plasma is concentrated. In contrast to the cartoon proposed by Cliver et al. (1986) that places the filament material at the centre of the cavity, the filament material is located at the bottom of the cavity, which is more consistent with the model of Martens \& Kuin (1989).

In the observations reported here, magnetic curvature compensates for gravity to create an equilibrium state where the density is considerably increased at the bottom of the cavity. We also show that the flows along field lines and varying from one wavelength to another are important for the initiation (Fig. 2b) and relaxation (Fig. 2d) phases of the eruption. We also note that the rise of the cavity (divided in two stages) is similar to the plasmoid eruption initiated by an impulsive flare as reported by Ohyama et al. (1997; see also Shibata 1998).

This preliminary study describes the structure and evolution of a polar crown filament and its cavity projected onto the plane of the sky and, in any case, gives a full picture of the eruption process. In a forthcoming paper, we will discuss the possible triggers of the cavity eruption of concomitant external (flares, CMEs) and internal (kink/torus instability, mass loading) phenomena by combining SDO/AIA and STEREO/SECCHI/EUVI images, which give us a more realistic $3 \mathrm{D}$ representation of the event.

Acknowledgements. We thank the referee for his/her useful comments helping to improve the letter. The SDO/AIA data have been obtained through the University of Central Lancashire database. The data used are provided courtesy of NASA/SDO and the AIA science team. CEA acknowledges the support of the STFC studentship programme. The SOHO/LASCO CME catalog is generated and maintained at the CDAW Data Center by NASA and The Catholic University of America in cooperation with the Naval Research Laboratory. SOHO is a project of international cooperation between ESA and NASA.

\section{References}

Carmichael, H. 1964, in AAS-NASA Symp. on Physics of Solar Flares, 451 Cliver, E. W., Dennis, B. R., Kiplinger, A. L., et al. 1986, ApJ, 305, 920 Forbes, T. G., Linker, J. A., Chen, J., et al. 2006, Space Sci. Rev., 123, 251 Gibson, S. E., Foster, D., Burkepile, J., de Toma, G., \& Stanger, A. 2006, ApJ, 641,590

Gibson, S. E., Kucera, T. A., Rastawicki, D., et al. 2010, ApJ, 724, 1133

Habbal, S. R., Druckmüller, M., Morgan, H., et al. 2010, ApJ, 719, 1362

Hirayama, T. 1974, Sol. Phys., 34, 323

Hudson, H., \& Schwenn, R. 2000, Adv. Space Res., 25, 1859

Hudson, H. S., Acton, L. W., Harvey, K. L., \& McKenzie, D. E. 1999, ApJ, 513, L83

Illing, R. M. E., \& Hundhausen, A. J. 1986, J. Geophys. Res., 91, 10951

Kopp, R. A., \& Pneuman, G. W. 1976, Sol. Phys., 50, 85

Lemen, J. R., Title, A. M., \& Akin, D. J. E. 2011, Sol. Phys.

Lin, J. 2002, Chin. J. A\&A, 2, 539

Martens, P. C. H., \& Kuin, N. P. M. 1989, Sol. Phys., 122, 263

O’Dwyer, B., Del Zanna, G., Mason, H. E., Weber, M. A., \& Tripathi, D. 2010, A\&A, 521, A21

Ohyama, M., Shibata, K., Yokoyama, T., \& Shimojo, M. 1997, Adv. Space Res., 19,1849

Shibata, K. 1998, Ap\&SS, 264, 129

Sturrock, P. A. 1968, in Structure and Development of Solar Active Regions, IAU Symp., 35, 471

Tsuneta, S. 1997, ApJ, 483, 507 